$$
n_{3}(t \rightarrow \infty)=\frac{\mathrm{I}_{s} \mathbf{T}_{p}\left(1-e^{-\sigma_{s} n d}\right)}{\beta_{3}\left[\beta_{2}+\mathrm{I}_{p}\left(q+q^{\prime}\right)\right]} .
$$

It is possible to distinguish between two distinct QC cases: Case I where the output transition occurs between levels $3 \rightarrow 1$, and Case II where the output transition occurs between levels $3 \rightarrow 2$. For Case I, the output flux is $q \beta_{3} n_{3}$, and this flux normalized to the signal flux $\Gamma_{s}$ is the quantum efficiency $\eta_{f}$ :

$$
\eta_{f}=\frac{q\left(1-e^{-\sigma_{s} n d}\right)}{\left(q+q^{\prime}\right)+\hbar \omega_{p} \ln 2 / \tau_{2} \sigma_{p} P_{p}}
$$

and where for all possible parameters $\eta_{f}<1$. For Case II, the output flux is $\left(1-q-q^{\prime}\right) \beta_{3} n_{3}$ and the steady-state quantum efficiency $\eta_{f}$ is

$$
\eta_{f}=\frac{\left(1-q-q^{\prime}\right)\left(1-e^{-\sigma_{n} n d}\right)}{\left(q+q^{\prime}\right)+\hbar \omega_{p} \ln 2 / \tau_{2} \sigma_{p} P_{p}}
$$

and $\eta_{f}$ can be greater than one.

The $\mathrm{QC}$ rise time is defined as the time required for the output flux to reach 90 per cent of the steady-state flux for a square leading edge signal pulse. Using (14), we find the rise time $\tau_{r}$ is given by the eq.

$$
e^{\alpha \tau_{r}}=10\left\{\cosh \Gamma \tau_{r}+\frac{\alpha}{\Gamma} \sinh \Gamma \tau_{r}\right\}
$$

where $\alpha$ and $\Gamma$ are dependent on pump power. The $\tau_{r}$ satisfying this equation for a given pump power can be found graphically.

In the region where $\Gamma_{p}$ is large enough to saturate the value of $\eta_{f}$, the expression of (20) can be simplified to

$$
e^{\left(q+q^{\prime}\right)\left(\tau_{r} \ln 2 / \tau_{3}\right)} \approx 10
$$

or

$$
\tau_{\mathrm{r}}=\frac{3.26 \tau_{3}}{q+q^{\prime}}
$$

An expression identical to $(20)$ is found for the fall time $\tau_{f}$ when the signal flux is abruptly shut off.

\section{REFERENCES}

[1] Bloembergen, N., presented at the 1958 Symp. on New Techniques in Microwave and Radio-Frequency Physics, Nat'l Acad. of Sciences, Washington, D. C.

[2] —, Phys. Rev. (Lett.), vol 2, 1959, p 84

[3] Shimoda, K., J. Phys. Soc. Japan (Notes), vol 14, 1959, p 966.

[4] Gelinas, R. W., Infrared detection by ideal irasers and narrow band counters, Rept No P-1844, The Rand Corp., Santa Monica, Calif., Oct 1959

[5] Porter, J. F., Jr., J. Appl. Phys., vol 32, 1961, p 825

[6] - Phys. Rev. (Lett.), vol 7, 1961, p 414.

[7] Brown, M. R., and W. A. Shand, Phys. Rev. (Lett.), vol 11, $1963, p 366$.

[8] , Phys. Rev. (Leth.), vol 12, 1964, p 367.

[9] Halsted, R. E., El R. Apple, and J. S. Prener, Phys. Rev. (Lett), vol 1, 1958, p 134

[10] Thys. Rev. (Lett.), vol 2, 1959, p 420 .

[11] Judd, B. R., Phys. Rev., vol 127, 1962, p 750.

[12] Ofelt, G. S., J. Chem. Phys., vol 37, 1962, p 511.

[13] Axe, J. D., Jr., J. Chem. Phys., vol 39, 1963, p 1154.

[14] Kisliuk, P., W. F. Krupke, and J. B. Gruber, J. Chem. Phys. vol $40,1964, \mathrm{p} 3606$.

[15] Gruber, J. B., W. F. Krupke, and J. M. Poindexter, J. Chem. Phys., vol 41, 1964, p 3363.

[16] Krupke, W. F., Rept No TDR-269(4250-25)-2, Aerospace Corp., El Segundo, Calif., Oct 1964.

17] RCA Photomultiplier Tube Data Sheet 7265-9-58.

[18] RCA Photomultiplier Tube Data Sheet 7102-12-57.

[19] Covit, B. Infrared detectors, Space/Aeronautics Mag., Nov 1961.

20] Jelley, J. V., J. Appl. Phys., vol 31, 1960, p 1145.

[21] Melngailis, I., A. J. Strauss, and R. H. Rediker, Semiconductor diode masers of ( $\left(n_{x} G a_{i-x}\right) A s$, Proc. IEEE (Correspondence), vol 51 , Aug 1963, pp 1154-1155.

[22] MeClure, D. S., and Z. Kiss, J. Chem. Phys., vol 39, 1963, p 3251.

23] Kaiser, W., G. B. Garrett, and D. L. Wood, Phys. Rev., vol 123, $1961, p 760$

[24] RCA Data Sheet for $p$-Type Au:Ge Detector, 1964.

[25] Carr, W. N., and G. E. Pittman, Appl. Phys. Lett., vol 3, 1963, p 173 .

\title{
Quantum Theory for Parametric Interactions of Light and Hypersound
}

\author{
A. YARIV, MEMBER, IEEE
}

\begin{abstract}
The problem of energy exchange between two electromagnetic modes of different frequencies and an acoustic mode is formulated and solved. The results of the quantum mechanical analysis are also analyzed in classical terms and are found to be consistent with the theory for parametric interactions. Specific cases treated include: parametric amplification of light, stimulated Brillouin scattering, and frequency conversion.
\end{abstract}

Manuscript received February 19, 1965.

The author is with the California Institute of Technology, Pasadena, Calif.

\section{INTRODUCTION}

$\prod$ N THIS PAPER we consider the general case of nonlinear interactions involving electromagnetic (EM) and sound waves. The treatment is quantum mechanical, but the final "working" equations can, also, be considered as classical.

The formalism developed here constitutes an extension of previous work by Louisell, Yariv, and Siegman [1] 
describing parametric interactions within an ensemble of EM waves.

A Boson quantization for both the EM and sound waves is used to obtain a set of differential equations which describe the coherent interaction of an arbitrary number of modes. These equations are solved for the case of two EM waves interacting with one sound wave. The first case discussed is that of an intense EM wave with a (radian) frequency $\omega_{p}$ in the presence of a sound wave $\omega_{s}$ and an "idler" EM wave $\omega_{i}$, where $\omega_{p}=\omega_{i}+\omega_{s}$. This case, which will be referred to as parametric amplification, includes as special cases: a) Parametric amplification of the idler wave, b) Parametric amplification of sound, c) Spontaneous oscillation of the sound and idler waves (Stimulated Brillouin Scattering [2], [3]), d) Generation of an idler wave by amplifying the energy, measured in number of quanta, of an input sound wave and, e) The reverse of $d$ ).

The second case discussed is one where the intense EM pump wave corresponds to the intermediate $\omega_{p}=\omega_{i}-\omega_{s}$ frequency. This leads to periodic energy exchange between the sound wave and the EM wave at $\omega_{i}$. In contrast to the first case the average number of quanta is not amplified.

The equations of motion, in the Heisenberg representation, for the creation and annihilation operators are solved for the case of no losses. These are used to obtain the expectation values of the various number of quanta operators. In the classical treatment we merely consider these operators as classical, normal-mode variables. The equations are in the form of the coupled-mode equations which have been found most suitable for treating parametric interactions [4]. Losses are added phenomenologically to obtain the threshold condition for parametric oscillation.

\section{Quantization of the Elegctromagneticc Field}

We assume that the electromagnetic field is confined within a closed surface $S$ of infinite conductivity and arbitrary shape. The dielectric medium is assumed lossless (the case of a lossy medium will be discussed in Section VIII). The total electric field $\mathrm{E}(\mathbf{r}, t)$ and magnetic field $\mathbf{H}(\mathbf{r}, t)$ are expanded in terms of Slater's modes ${ }^{5} E_{l}(\mathbf{r}, t)$ and $\mathbf{H}_{l}(\mathrm{r}, t)$, respectively

$$
\begin{aligned}
& \mathbf{E}(\mathbf{r}, t)=-\sum_{l} \frac{1}{\sqrt{\epsilon}} p_{l}(t) \mathbf{E}_{l}(\mathbf{r}) \\
& \mathbf{H}(\mathbf{r}, t)=\sum_{l} \frac{1}{\sqrt{\mu}} \omega_{l} q_{l}(t) \mathbf{H}_{l}(\mathbf{r})
\end{aligned}
$$

$\epsilon$ and $\mu$ have their conventional definitions, mks units are employed throughout. The eigen vector functions obey

$$
\begin{aligned}
& \nabla \times \mathbf{E}_{l}=k_{l} \mathbf{H}_{l}, \quad \nabla \times \mathbf{H}_{l}=k_{l} \mathbf{E}_{l} \\
& \mathbf{E}_{l} \times \mathbf{n}=\mathbf{H}_{l} \times \mathbf{n}=0 \text { on } S .
\end{aligned}
$$

It follows from (3) that

$$
\int_{V} \mathbf{E}_{l} \cdot \mathbf{E}_{m} d V=\int_{V} \mathbf{H}_{l} \cdot \mathbf{H}_{m} d V=0 \quad \text { if } \quad l \neq m .
$$

The functions are normalized according to

$$
\int_{V} \mathbf{E}_{l} \cdot \mathbf{E}_{m} d V=\int_{V} \mathbf{H}_{l} \cdot \mathbf{H}_{n_{t}} d V=\delta_{l, m} .
$$

Substitution of (1) and (2) into Maxwell equations yields

$$
\frac{d^{2} q_{l}}{d t^{2}}+\omega_{l}^{2} q_{l}=0
$$

and similar equation for $p_{l}$. The constant $k_{l}$ appearing in (3) is identified as $k_{l}=\omega_{l} \sqrt{\mu \epsilon}$. The total EM field Hamiltonian is given by

$H=\mu \int_{V} H^{2} d V+\epsilon \int_{V} E^{2} d V=\sum_{l}\left(p_{l}^{2}+\omega_{l}^{2} q_{l}^{2}\right)$

where use has been made of (1), (2), and (5). Considered as conjugate hermitian operators, $p_{l}$ and $q_{l}$ obey the commutation relations

$$
\left[p_{l}, p_{m}\right]=\left[q_{l}, q_{m}\right]=0 ; \quad\left[q_{l}, p_{m}\right]=i \hbar \delta_{l, m} .
$$

The creation operator $a_{l}^{+}$and its hermitian adjoint the annihilation operator $a_{l}$ are defined through

$$
\begin{aligned}
& q_{l}(t)=\left(\frac{\hbar}{2 \omega_{l}}\right)^{1 / 2}\left[a_{l}^{+}(t)+a_{l}(t)\right] \\
& p_{l}(t)=i\left(\frac{\hbar \omega_{l}}{2}\right)^{1 / 2}\left[a_{l}^{+}(t)-a_{l}(t)\right]
\end{aligned}
$$

so that

$$
a_{l}^{+}=\left(\frac{1}{2 \hbar \omega_{l}}\right)^{1 / 2}\left(\omega_{l} q_{l}-i p_{l}\right)
$$

Substituting (9) in (8) gives the commutation relations

$$
\begin{aligned}
& {\left[a_{l}, a_{m}\right]=\left[a_{l}^{+}, a_{m}^{+}\right]=0} \\
& {\left[a_{l}(t), a_{m}^{+}(t)\right]=\delta_{l m} .}
\end{aligned}
$$

In terms of a $a_{l}$ and $a_{l}^{+}$the field Hamiltonian (7) becomes

$$
H_{0}=\sum_{l} \hbar \omega_{l}\left(a_{l}^{+} a_{l}+\frac{1}{2}\right) \text {. }
$$

The operators $a_{l}, a_{l}^{+}$are in the Heisenberg representation. Their time evolution is given by

$$
\frac{d a_{l}}{d t}=\frac{1}{i \hbar}\left[a_{l}, H_{0}\right]=-i \omega_{l} a_{l}
$$

so that

$$
a_{l}(t)=a_{\imath 0} e^{-i \omega t t}
$$

$a_{l}^{+}$is, likewise, given by

$$
a_{l}^{+}(t)=a_{l 0}^{+} e^{+i \omega_{l} t} .
$$

Denoting by $\left|n_{20}\right\rangle$ the set of eigenfunctions, of the operators $a_{l 0}^{+} a_{20}$ we have

$$
\begin{aligned}
& a_{l 0}^{+}\left|n_{l 0}\right\rangle=\left(n_{l 0}+1\right)^{1 / 2}\left|n_{l 0}+1\right\rangle \\
& a_{l 0}\left|n_{l 0}\right\rangle=\left(n_{l 0}\right)^{1 / 2}\left|n_{l 0}-1\right\rangle
\end{aligned}
$$

and

$$
a_{l 0}^{+} a_{l 0}\left|n_{l 0}\right\rangle=n_{l 0}\left|n_{l 0}\right\rangle
$$


so that, in view of (11), $n_{70}$ is the number of quanta, ignoring zero field vibrations, in the $l$ th mode at $t=0$. For the case described above for which (13) apply, we have $a_{l}^{+}(t) a_{l}(t)=a_{l 0}^{+} a_{l 0}$ so that the excitation level (number of quanta) in each mode is a constant of the motion. In Section IV we will introduce the coupling between modes and the excitation of the individual modes will be time dependent.

\section{Quantization of the Acoustic Field}

We assume a homogeneous isotropic medium of mass density $\rho$, of length $L$, and bulk modulus (elastic stiffness constant) $T$, let the deviation of a point $x$ from its equilibrium position be given by $q(x, t)$. The strain along the $x$ direction is then given by $\partial g / \partial x$. The Lagrangian density $\alpha$ is [6]

$$
\alpha=\frac{1}{2} \rho\left(\frac{\partial q}{\partial t}\right)^{2}-\frac{1}{2} T\left(\frac{\partial q}{\partial x}\right)^{2} .
$$

The momentum canonically conjugate to $q$ is

$$
p=\frac{\partial \alpha}{\partial \dot{q}}=\rho \dot{q} .
$$

The Hamiltonian density $\mathfrak{F C}=p \dot{q}-\alpha$ becomes

$$
\mathfrak{H C}=\frac{p^{2}}{2 \rho}+\frac{1}{2} T\left(\frac{\partial q}{\partial x}\right)^{2} .
$$

The operator $q(x, t)$ can be expanded in a Fourier series as

$$
q(x, t)=L^{-1 / 2} \sum_{k=-\infty}^{+\infty} Q_{k}(t) e^{i k \cdot x}
$$

where

$$
Q_{k}(t)=L^{-1 / 2} \int_{-L / 2}^{+L / 2} q(\xi, t) e^{-i k \xi} d \xi .
$$

From the hermiticity of $q(x, t)$ it follows that

$$
Q_{k}=\left(Q_{-k}\right)^{+}
$$

so that the $Q_{k}{ }^{\prime}$ 's are not hermitian operators.

In a similar manner we introduce $P_{k}=\left(P_{-k}\right)^{+}$through

$$
P_{k}=L^{-1 / 2} \int_{-L / 2}^{+L / 2} p(\xi) e^{+i k \xi} d \xi
$$

so that

$$
p(x)=L^{-1 / 2} \sum_{k} P_{k} e^{-i k x} .
$$

The total Hamiltonian is $H=\int$ Je $d V$.

Substituting (18) and (19) in (17), carrying out the integration, and making use of the closure property of $e^{i k x}$ gives

$$
H=\sum_{k=-\infty}^{+\infty}\left(\frac{1}{2 \rho} P_{k} P_{-k}+\frac{1}{2} T k^{2} Q_{k} Q_{-k}\right) A
$$

where $A$ is the cross-sectional area of the acoustic wave normal to $x$. The $\sqrt{A} Q_{k}$ 's and $\sqrt{A} P_{k}$ 's obey commuta- tion relations identical to (8). The only noncommuting pair is $\left(Q_{k}, P_{k}\right)$ for which

$$
A\left[Q_{k}, P_{k^{\prime}}\right]=i \hbar \delta_{k, k^{\prime}}
$$

The annihilation and creation operators for the phonon field are introduced by

$$
a_{k}^{+}=-i\left(\frac{A}{2 \rho \omega_{k} h}\right)^{1 / 2} P_{k}+\left(\frac{T A}{2 \hbar \omega_{k}}\right)^{1 / 2}|k| Q_{-k}
$$

and its hermitian adjoint

$$
a_{k}=i\left(\frac{A}{2 \rho \omega_{k} \hbar}\right)^{1 / 2} P_{-k}+\left(\frac{T A}{2 \hbar \omega_{k}}\right)^{1 / 2}|k| Q_{k}
$$

$\omega_{k}=(T / \rho)^{\frac{1}{2}}|k|=v_{0} k$ where $v_{0}$ is the velocity of sound in the medium. Expressing $P_{k}$ and $Q_{k}$ in terms of $a_{k}$ and $a_{k}^{+}$gives

$$
\begin{aligned}
P_{k} & =i\left(\frac{\hbar \omega_{k} \rho}{2 A}\right)^{1 / 2}\left(a_{k}^{+}-a_{-k}\right) \\
Q_{k} & =\left(\frac{\hbar \omega_{k}}{2 T A}\right)^{1 / 2} \frac{1}{|k|}\left(a_{k}^{+}+a_{-k}\right) .
\end{aligned}
$$

It follows from (22) and (23) that the only noncommuting pair of operators is, again, $a_{k}, a_{k}^{+}$for which

$$
\left[a_{k}, a_{k}^{+},\right]=\delta_{k, k} \cdot
$$

Using (24) and (25) in (21) leads to

$$
H_{\mathrm{acoustic}}=\sum_{k} \hbar \omega_{k}\left(a_{k}^{+} a_{k}+\frac{1}{2}\right) .
$$

The operators $a_{k}^{+}$and $a_{k}$ are the time-dependent (Heisenberg representation) creation and annihilation operators for the phonon modes. Their properties are described by equations identical to (14).

\section{The Interaction Between Modes}

In the absence of nonlinear interactions the total Hamiltonian, including both acoustic and electromagnetic modes, is given by

$$
H_{0}=\sum_{l} \hbar \omega_{l}\left(a_{l}^{+} a_{l}+\frac{1}{2}\right)+\sum_{k} \hbar \omega_{k}\left(a_{k}^{+} a_{k}+\frac{1}{2}\right)
$$

where the $l$ and $k$ denote summation over electromagnetic and acoustic modes, respectively. We have already shown, in Section II, that under these conditions the operators are given by $a_{k}^{+}=a_{k 0}^{+} \exp \left(i \omega_{k} t\right)$ so that $\left\langle a_{k}^{+}(t) a_{k}(t)\right\rangle=$ $\left\langle a_{k}^{+}(0) a_{k}(0)\right\rangle$. The number of quanta in each mode is, thus, time invariant and no energy exchange takes place.

The interaction between modes is introduced in a manner suggested by Louisell et al. [1]. In that work it was shown that a time varying dielectric constant can provide the necessary coupling between modes. This variation was introduced explicitly into the formalism. In our case the dielectric modulation is introduced implicitly by allowing the dielectric constant to depend on the local strain $\partial q / \partial x$. The acoustic vibrations can thus be viewed as causing the time and space variation of the dielectric constant which in turn couples the EM waves. 
We assume that the strain $\partial q / \partial x$ and the resulting dielectric modulation $\delta \epsilon$ are related by

$$
\delta \epsilon=-\gamma \frac{\partial q}{\partial x}
$$

$\gamma$ can be identified with the electrostrictive coefficient of ref. 2 by noting that the fractional density variation $\delta \rho$ is given by $\delta \rho / \rho=-\partial q / \partial x$. The perturbation Hamiltonian $H^{\prime}$ is given, as in Louisell et al., by the added stored electromagnetic energy $\frac{1}{2} \int \delta \epsilon E^{2} d V$ resulting from the change in $\epsilon$.

$$
H^{\prime}=\frac{-\gamma}{2} \int_{V} \frac{\partial q}{\partial x} E^{2} d V .
$$

According to (18a)

$$
\frac{\partial q}{\partial x}=L^{-1 / 2} \sum_{k=-\infty}^{+\infty} i k Q_{k} e^{i k x} .
$$

Using (24) and reversing the order of summation for the terms involving $a_{-k}$ results in

$\frac{\partial g}{\partial x}=V^{-1 / 2} \sum_{k=-\infty}^{+\infty}(i k)\left(\frac{\hbar \omega_{k}}{2 T}\right)^{1 / 2}\left(\frac{1}{|k|}\right)\left(a_{k} e^{i k x}-a_{k}^{+} e^{-i k x}\right)$

where $V=L A$.

Substituting (30) in (29), making use of (1), (5), and (9) and carrying out the integration yields

$$
\begin{aligned}
H^{\prime}=\frac{-\gamma}{2} \int_{V} & {\left[V^{-1 / 2} \sum_{k=-\infty}^{+\infty}(i k)\left(\frac{\hbar \omega_{k}}{2 T}\right)^{1 / 2}\left(\frac{1}{|k|}\right)\right.} \\
& \cdot\left(a_{k} e^{+i k x}-a_{k}^{+} e^{-i k x}\right) \sum_{l} \sum_{l^{\prime}} \frac{\epsilon^{-1} \hbar}{2}\left(\omega_{l} \omega_{l^{\prime}}\right)^{1 / 2} \\
& \left.\cdot\left(a_{l}^{+}-a_{l}\right)\left(a_{l}^{+},-a_{l}\right) \mathbf{E}_{l}(\mathbf{r}) \cdot \mathbf{E}_{l^{\prime}}(\mathbf{r})\right] d V .
\end{aligned}
$$

In terms of the coupling coefficient

$\kappa_{l, l, k>0}=\frac{-i \gamma}{4 \epsilon}\left(\frac{\hbar^{3}}{2 V T}\right)^{1 / 2}\left(\omega_{l} \omega_{l} \cdot \omega_{k}\right)^{1 / 2} \int_{V} e^{i k x} \mathbf{E}_{l}(\mathbf{r}) \cdot \mathbf{E}_{l},(\mathbf{r}) d V$

and

$\kappa_{l, l^{\prime},-k}=\left(\kappa_{l, l^{\prime}, k}\right)^{*}$.

Equation (31) can be rewritten as

$$
\begin{array}{r}
H^{\prime}=\sum_{l} \sum_{l^{\prime}} \sum_{k}\left[\kappa_{l, l^{\prime}, k} a_{k}\left(a^{+}{ }_{l}-a_{l}\right)\left(a_{l^{\prime}}{ }^{+}-a_{l^{\prime}}\right)\right. \\
\left.+\left(\kappa_{l, l^{\prime}, k}\right)^{*} a_{k}^{+}\left(a_{l}^{+}-a_{l}\right)\left(a_{l^{+}}^{+}-a_{l^{\prime}}\right)\right] .
\end{array}
$$

The total Hamiltonian is $H_{T}=H_{0}+H^{\prime}$ where $H_{0}$ is given by (27).

The equations of motions for the boson operators can be written directly by using (12) with $H_{0}$ replaced by $H_{0}+H^{\prime}$. The result is

$$
\begin{aligned}
\frac{d a_{i}^{+}}{d t}=i \omega a_{i}^{+}-i \sum_{k} \sum_{l} & {\left[\frac{\kappa_{i, l, k}}{\hbar} a_{k}\left(a_{l}^{+}-a_{l}\right)\right.} \\
& \left.+\left(\frac{\kappa_{i, l, k}}{\hbar}\right)^{*} a_{k}^{+}\left(a_{l}^{+}-a_{l}\right)\right]
\end{aligned}
$$

so that in general each mode $j$ is "coupled" into all the other modes. Fortunately we can reduce the "hopeless" case represented by (34) to cases involving a small number of interacting modes without sacrificing physical realism. We notice, first, that the perturbation contribution within the double summation sign, includes terms oscillating at radian frequencies $\pm \omega_{k} \pm \omega_{l}$. The nonperturbed term oscillates at a frequency of $\omega_{j}$. It follows that synchronous contribution can arise only from perturbation terms where $\pm \omega_{k} \pm \omega_{l}=\omega_{j}$. The contribution of other terms gives rise to rapidly fluctuating terms which average out to zero over time intervals of interest. As pointed out by Louisell et al., the argument presented above is equivalent to retaining only the secular part of the Hamiltonian, thus conserving energy. A further reduction of the number of interacting modes is brought about through the coupling coefficient $\kappa_{i, l, k}$. As will be shown in Section X, $\kappa_{i, l, k}$ is generally very small except for special cases in which phase matching takes place.

\section{Three Mode Interaction}

The remainder of this paper will deal with cases where the interaction is limited to three modes only. Two of the modes will be taken as electromagnetic and the third mode as acoustic. Adopting the convention of parametric interaction we will designate one EM mode, usually the intense input mode, as the pump and take its frequency as $\omega_{p}$. The second EM mode is the " $i$ " idler mode with a frequency $\omega_{i}$. The acoustic mode will be designated as the " $s$ " sound, or signal mode oscillating at $\omega_{z}$.

The Hamiltonian for the three modes takes the simple form

$$
\begin{aligned}
H_{T}=\hbar \omega_{p}\left(a_{p}^{+} a_{p}+\frac{1}{2}\right)+ & \hbar \omega_{i}\left(a_{i}^{+} a_{i}+\frac{1}{2}\right)+\hbar \omega_{s}\left(a_{s}^{+} a_{s}+\frac{1}{2}\right) \\
+ & +\kappa a_{s}\left(a_{p}^{+}-a_{p}\right)\left(a_{i}^{+}-a_{i}\right) \\
& +\kappa^{*} a_{s}^{+}\left(a_{p}^{+}-a_{p}\right)\left(a_{i}^{+}-a_{i}\right)
\end{aligned}
$$

where $\kappa=\kappa_{p, i, s}$.

The equations of motion (34) reduce to

$$
\begin{aligned}
& \frac{d a_{p}^{+}}{d t}=i \omega_{p} a_{p}^{+}-\frac{i \kappa}{\hbar} a_{s}\left(a_{i}^{+}-a_{i}\right)-\frac{i \kappa^{*}}{\hbar} a_{s}^{+}\left(a_{i}^{+}-a_{i}\right) \\
& \frac{d a_{i}^{+}}{d t}=i \omega_{i} a_{i}^{+}-\frac{i \kappa}{\hbar} a_{s}\left(a_{p}^{+}-a_{p}\right)-\frac{i \kappa^{*}}{\hbar} a_{s}^{+}\left(a_{p}^{+}-a_{p}\right)
\end{aligned}
$$

(plus their hermitian adjoints) for the optical modes. The equations of motion for the acoustic mode $a_{s}^{+}$is derived from (35) and (12) as

$$
\frac{d a_{s}^{+}}{d t}=i \omega_{s} a_{s}^{+}+\frac{i_{\kappa}}{\hbar}\left(a_{p}^{+}-a_{p}\right)\left(a_{i}^{+}-a_{i}\right)
$$

Before proceeding with detailed discussions of the solutions we assume that one of the two optical modes, arbitrarily chosen as the $p$ mode, is so intense that it can be described classically. Furthermore, it is assumed that the amount of energy lost or gained by this mode 
to the idler $i$ and acoustic $s$ modes is small compared to its own stored energy, so that its amplitude can be taken as a constant. This assumption conforms closely to situations prevailing in many experiments, especially thase in which the pump power is provided by an intense laser source.

If we take the electric field of the pump mode as $E_{p 0}$ $\cos \omega_{p} t \mathrm{E}_{p}(\mathbf{r})$, we can establish through (1) and (9) the identity: $\left(a_{p}^{+}-a_{p}\right)=+i\left(\epsilon / 2 \hbar \omega_{p}\right)^{\frac{1}{2}} E_{p 0}\left(e^{i \omega_{p} t}+e^{-i \omega_{p} t}\right)$. Using it in (36) and (37) results in

$\frac{d a_{i}^{+}}{d t}=i \omega_{i} a_{i}^{+}-i \eta\left(e^{i \omega_{p} t}+e^{-i \omega_{p} t}\right) a_{s}+i \eta^{*}\left(e^{i \omega_{p} t}+e^{-i \omega_{p} t}\right) a_{s}^{+}$

$\frac{d a_{s}^{+}}{d t}=i \omega_{s} a_{s}^{+}+i \eta\left(e^{i \omega_{p} t}+e^{-i \omega_{p} t}\right)\left(a_{i}^{+}-a_{i}\right)$

where the new coupling coefficient $\eta$ is defimed by

$\eta=\frac{\gamma}{(64 \epsilon T)^{1 / 2}}\left(\omega_{i} \omega_{s}\right)^{1 / 2} E_{p 0} \int_{V} V^{-1 / 2} e^{i k_{s} x} \mathrm{E}_{p}(\mathrm{r}) \cdot E_{i}(\mathbf{r}) d V$.

Equation (38), together with its hermitian adjoint, is the main working equation for treating nonlinear interactions between sound and EM waves. From this equation we will obtain the differential equations obtained in specific situations. The case of parametric amplification and oscillation of a sound wave at $\omega_{s}$ and an EM wave at $\omega_{i}$ through pumping by an EM wave at $\omega_{p}=\omega_{i}+\omega_{s}$ will be discussed in Section VI. The ease of mixing of a sound wave $\omega_{s}$ with an EM pump wave $\omega_{p}$ to generate an EM wave at $\omega_{i}=\omega_{p}+\omega_{s}$ will be considered in Section IX. The inclusion of loss will be discussed in Section VII.

\section{Parametric Amplification and Oscillation-Classical Treatment}

We consider the interaction between an intense EM wave at $\omega_{p}$, a sound wave at $\omega_{s}$ and a second EM wave at $\omega_{i}$. We take $\omega_{p}=\omega_{i}+\omega_{s}$. Retaining only the synchronous terms in (38), i.e., those having the same time dependence as $a_{i}^{*}$ and $a_{s}^{+}$, we get

$$
\begin{aligned}
& \frac{d a_{i}^{*}}{d t}=i \omega_{i} a_{i}^{+}-i \eta e^{i \omega_{p} t} a_{s} \\
& \frac{d a_{s}}{d t}=-i \omega_{s} a_{s}+i \eta^{*} e^{-i \omega_{p} z} a_{i}^{*}
\end{aligned}
$$

and their hermitian adjoints.

These equations are identical to the classical parametric amplifier equations [4] if the creation and annihilation operators are identified with the classical mode amplitudes and their complex conjugates. They were also obtained by Louisell et al. [1], for the case of two EM modes coupled through dielectric modulation.

It follows from (40) and (41) that

$$
\frac{d}{d t}\left(a_{i}^{+} a_{i}\right)=\frac{d}{d t}\left(a_{s}^{+} a_{s}\right)
$$

This means that the number of idler photons created in a given time is equal to the number of phonons created during the same period. This statement when expressed in terms of powers in just the Manley-Rowe relation $P_{i} / \omega_{i}=P_{s} / \omega_{s}$, since the energy in any mode, say $j$ is given by $\left(a_{j}^{+} a_{j}\right) \hbar \omega_{j}$.

The solutions of (40) and (41) are

$$
\begin{aligned}
& a_{s}(t)=e^{-i \omega_{s} t}\left(a_{s 0} \cosh \eta t+i a_{i 0}^{+} \sinh \eta t\right) \\
& a_{i}^{+}(t)=e^{+i \omega_{i} t}\left(a_{i 0}^{+} \cosh \eta t-i a_{s 0} \sinh \eta t\right) .
\end{aligned}
$$

For the purpose of a classical analysis we can regard (42) and (43) as the solutions of the normal mode amplitudes where the energy per mode is given by $\hbar \omega_{s} a_{i}^{+} a_{s}$ and $\hbar \omega_{i} a_{i}^{+} a_{i}$. It is more convenient, however, to deal even classically, with the number of quanta (photons and phonons) per mode which is given simply by $a^{+} a$. The number of phonons at $\omega_{s}$ at $t=0$ is thus given by $n_{s 0}=$ $a_{s 0}^{*} a_{s 0}$ ( $a_{3}^{+}$becomes $a^{*}$ in the classical treatment), while the number of photons present, initially, at the idler mode is $n_{i 0}=a_{i 0}^{*} a_{i 0}$. Solving for $n_{s}(t)=a_{s}^{*}(t) a_{s}(t)$ and for $n_{i}(t)$ results in

$$
\begin{aligned}
n_{s}(t)_{\mathrm{classical}}=n_{s 0} \cosh ^{2} \eta t & +n_{i 0} \sinh ^{2} \eta t \\
& +\sinh 2 \eta t \operatorname{tm}\left(a_{i 0} a_{s 0}\right)
\end{aligned}
$$

and

$$
\begin{aligned}
n_{i}(t)_{\mathrm{elassical}}=n_{i 0} \cosh ^{2} \eta t & +n_{s 0} \sinh ^{2} \eta t \\
& +\sinh 2 \eta t \operatorname{Im}\left(a_{i 0} a_{s 0}\right)
\end{aligned}
$$

where $I m$ stands for the "imaginary part of."

The neglect of all losses limits, somewhat, the practical importance of these equations. Their usefulness is mainly in pointing out the basic parametric nature of the process and in describing the ultimate behavior under ideal conditions. Consider first, the case when at $t=0$ we have only a sound wave excited, i.e., $n_{i}(o)=0$. This will cause, according to (44) and (45), a buildup of the sound wave and the idler wave according to

$$
\begin{aligned}
& n_{s}(t)=n_{s 0} \cosh ^{2} \eta t \underset{\eta \rightarrow \gg 1}{\rightarrow} \frac{n_{s 0}}{4} e^{2 \eta t} \\
& n_{i}(t)=n_{s 0} \sinh ^{2} \eta i \underset{\eta t \gg 1}{\rightarrow} \frac{n_{s 0}}{4} e^{2 \eta t} .
\end{aligned}
$$

In a resonator type of analysis, which is the one we have employed, so far, (46) shows that oscillation at both $\omega_{i}$ and $\omega_{s}$ can be triggered by any residual mode energy $n_{s 0}$ at $t=0$. The lack of a threshold pumping level is, of course, due to our neglect of losses. This situation will be rectified in Section VIII. If we consider (46) as applying to a traveling wave type interaction we must replace $t$ by $x / v_{s}$ where $x$ is the distance traveled. Equation (46) shows how an input sound wave is amplified by a factor $\frac{1}{4} e^{2 \eta x / u_{s}}$ due to pumping by an EM wave at $\omega_{p}$. The concomitant spatial amplification of the idler EM wave is described by $n_{i}(x)=\left(n_{s} / 4\right) e^{2 \eta x / v s}$ so that we have a means 
of converting an input sound wave into an electromagnetic wave with an amplification of the number of input quanta. This makes it possible to detect the presence of phonons by optical measurements.

The case of no "input" phonons i.e., $n_{s}(o)=0$, leads to

$$
\begin{aligned}
& n_{s}(t)=n_{i 0} \sinh ^{2} \eta t \underset{\eta t \gg 1}{\longrightarrow} \frac{n_{i 0}}{4} e^{2 \eta t} \\
& n_{i}(t)=n_{i 0} \cosh ^{2} \eta t \underset{\eta t \gg 1}{\longrightarrow} \frac{n_{i 0}}{4} e^{2 \eta t} .
\end{aligned}
$$

In practice this condition is less likely to be encountered except at extremely low temperatures. The thermal excitation causes $n_{s}(o)$ to be of the order of $k T / \hbar \omega_{s}$, so that for microwave phonons we have a thermal excitation level corresponding to a few hundred acoustic quanta per mode at room temperature. As an amplifier of the idler radiation $\omega_{i}$, it follows from (45), that for linear amplification the temperature must be low enough so that the condition

$$
n_{i 0} \gg n_{s 0}=\left(e^{* \omega s / k T}-1\right)^{-1}
$$

is fulfilled.

\section{Parametric Amplification AND Oscrluation-QuaNtum-Mechanical Treatment}

If we consider the $a^{+1} \mathrm{~s}$ in (42) as creation operators and the $a^{\prime}$ 's as annihilation operators we can use them to obtain the expectation value of any function $F\left(a, a^{+}\right)$. We are interested, in particular, in the time evolution of the average number of quanta in each mode. The expectation values for the number of quanta are:

$$
\begin{aligned}
& n_{s}(t)=\left\langle\psi(0)\left|a_{s}(t)^{+} a_{s}(t)\right| \psi(0)\right\rangle \\
& n_{i}(t)=\left\langle\psi(o)\left|a_{i}^{+}(t) a_{i}(0)\right| \psi(0)\right\rangle
\end{aligned}
$$

where $\psi(o)$ is the initial wave function of the combined acoustic-idler system. We take $\psi(o)=\left|n_{i 0}, n_{s 0}\right\rangle$ which is a product of the respective harmonic oscillator wave functions with quantum numbers $n_{i 0}$ and $n_{s 0} . n_{i 0}$ and $n_{s 0}$ are, as in Section VI, the number of quanta present at $t=0$ at the idler and sound modes, respectively. In taking $\psi(0)=\left|n_{i 0}, n_{s 0}\right\rangle$ we specify the exact number of quanta in each mode and, because of the uncertainty principle, sacrifice all the phase information. This gives a result which represents an average over the relevant phases. This, still, leads to the correct result if we are only interested in the average number of quanta. In a more exact treatment we would use a coherent superposition of states for the initial wave-function $\psi(o)^{1,7,8}$. This aspect of the problem will not be treated here.

From (42) and (43) we have

$$
\begin{array}{r}
a_{s}^{+}(t) a_{s}(t)=a_{s 0}^{+} a_{s 0} \cosh ^{2} \eta t+\left(1+a_{i 0}^{+} a_{i 0}\right) \sinh ^{2} \eta t \\
+\frac{1}{2} i \sinh 2 \eta t\left[a_{s 0}^{+} a_{i 0}^{+}-a_{s 0} a_{i 0}\right]
\end{array}
$$

and a similar expression for $a_{i}^{+}(t) a_{i}(t)$ in which the subscripts $s$ and $i$ are interchanged. Substituting (50) in (48) and (49) and using (14a) and (14b) yields the following expression for the average number of quanta as a function of time

$$
\begin{gathered}
n_{s}(t)=\left\langle n_{i 0}, n_{s 0}\left|a_{s}^{+}(t) a_{s}(t)\right| n_{i 0}, n_{s 0}\right\rangle \\
=n_{s 0} \cosh ^{2} \eta t+\left(1+n_{i 0}\right) \sinh ^{2} \eta t \\
n_{i}(t)=n_{i 0} \cosh ^{2} \eta t+\left(1+n_{s 0}\right) \sinh ^{2} \eta t
\end{gathered}
$$

If we neglect the phase dependent part of (44) and (45) then the quantum mechanical solutions for the expectation value of the number of quanta $n_{s}(t)$ and $n_{i}(t)$ as given by (51) and (52) are identical to the classical solutions, (44) and (45) [1], except for the number unity appearing inside the bracket. This represents the zero field vibration energy present even when $n_{i 0}=n_{s 0}=0$. Viewing (51) and (52) as those of an amplifier, this term represents the noise added to the signal by the zero field vibrations. This noise is equivalent according to (51) and (52), to an effective noise input of one quantum per mode. This has been shown [1], [7] to be the limiting sensitivity of coherent amplifiers. The missing phase dependent term [last one in (45)] is due, as previously noted, to our choice of $\psi(0)=\left|n_{i 0}, n_{\mathrm{s} 0}\right\rangle$ and can be recovered by choosing a coherent (Poisson Packet) initial state. ${ }^{1}$

If we consider (51) and (52) as describing the buildup of the simultaneous idler and acoustic oscillations under pumping at $\omega_{p}$, we see that even at zero absolute temperature, i.e., when $n_{i 0}=n_{s 0}=0$, oscillation will build up due to zero field vibrations. It is of (dubious) academic interest to note that acoustic oscillation can be considered as initiated by the electromagnetic idler zero-field energy while the reverse is true for the idler mode oscillation.

\section{Parametric Amplification and Oscmllation in the Presence of Losses}

In Section VI we saw how "pumping" by an EM wave at $\omega_{p}$ can give rise to a simultaneous acoustic $\left(\omega_{s}\right)$ and electromagnetic $\left(\omega_{i}\right)$ oscillations where $\omega_{p}=\omega_{i}+\omega_{s}$. Since no losses were included, the pumping threshold for this oscillation was zero.

It can be shown that in the high $Q$ limit, i.e., when the energy lost per cycle is small compared to the stored energy, the effect of losses is merely to modify the first terms on the right side of (40) and (41) and not the cross coupling term. Or, if we express (40) and (41) in

1 It may help to recall here that the electric field intensity in the optical resonator was taken as

$$
\mathbf{E}_{p}(\mathbf{r}, t)=E_{p 0} \cos \omega_{p} t \mathrm{E}_{p}(\mathbf{r})
$$

where

$$
\int_{V} \mathrm{E}_{p}(\mathbf{r}) \cdot \mathrm{E}_{p}(\mathbf{r}) d V=\mathbf{1}
$$

for a $T E M$ type propagation along the $\mathbf{k}_{p}$ direction $E_{p}(\mathrm{r})$ can be taken as

$$
E_{p}(\mathrm{r})=\left(\frac{2}{V_{l}}\right)^{1 / 2} \sin \mathrm{k} \cdot \mathrm{r}
$$

so that the peak electric field is $\left(2 / V_{l}\right)^{\frac{1}{2}} E_{p 0}$. 
a matrix form as $d \tilde{a} / d t=\tilde{k} \tilde{a}$, the diagonal terms are the only ones affected. Equations (40) and (41) become

$$
\begin{aligned}
& \frac{d a_{i}^{+}}{d t}=+i \omega_{i} a_{i}^{+}-\frac{a_{i}^{+}}{T_{i}}-i \eta e^{i \omega_{p} t} a_{s} \\
& \frac{d a_{s}}{d t}=-i \omega_{s} a_{s}-\frac{a_{s}}{T_{s}}+i \eta^{*} e^{-i \omega_{p} t} a_{i}^{+}
\end{aligned}
$$

where $T_{i}$ and $T_{s}$ are the "ringing" times for the idler and sound modes, respectively, which are added phenomenologically. Substituting $a_{i}^{+}=A_{i}^{+} e^{+i \omega_{i} t}$ and $a_{s}=A_{s} e^{-i \omega_{s} t}$ in (53) leads to

$$
\begin{aligned}
& \frac{d A_{i}^{+}}{d t}=-\frac{A_{i}^{+}}{T_{i}}-i \eta A_{s} \\
& \frac{d A_{s}}{d t}=-\frac{A_{s}}{T_{s}}+i \eta^{*} A_{i}^{+} .
\end{aligned}
$$

Assuming $\exp (\alpha t)$ behavior for the variables in (54) leads to

$$
\alpha=\frac{-\left(\frac{1}{T_{s}}+\frac{1}{T_{i}}\right) \pm \sqrt{\left(\frac{1}{T_{s}}+\frac{1}{T_{i}}\right)^{2}+4\left(\eta \eta^{*}-\frac{1}{T_{i} T_{s}}\right)}}{2}
$$

for the exponential growth factor.

When the condition

$$
\eta \eta^{*} \geq \frac{1}{T_{i} T_{s}}
$$

if fulfilled, $\alpha \geq 0$. Both the acoustic and the idler wave will grow with time. This corresponds to what Chiao et al. [2], call stimulated Brillouin scattering. Using our point of view it is seen to represent the start-oscillation condition for a parametric oscillator. A similar expression was derived by Kingston [9] for the case of an optical parametric oscillator. Using the definition of $\eta$ (39) and the fact that $E_{p 0}$ is related to the peak electric field $E_{0}$ by $^{1} E_{0} \sim\left(2 / V_{l}\right)^{\frac{1}{2}} E_{p 0}$, the threshold condition (56) can be written as

$E_{0}^{2} \geq \frac{64 T_{\epsilon}}{\gamma^{2} L_{s} k_{s} L_{i} k_{i}}\left(\frac{V_{s}}{V_{l}}\right)\left(\int_{V} e^{i k_{s} \cdot \mathrm{r}} \mathrm{E}_{p}(\mathrm{r}) \cdot \mathrm{E}_{i}(\mathrm{r}) d V\right)^{-2}$

where $L_{i}=c T_{i}$ and $L_{s}=v_{0} T_{s}$ are the mean distances traveled during a lifetime by an idler photon and a phonon, respectively.

$V_{l}$ and $V_{s}$ are the volumes of the EM modes and acoustic modes respectively. For minimum threshold the factor

$$
\int_{V} e^{i k r_{0} \cdot \mathbf{r}} \mathbf{E}_{p}(\mathbf{r}) \cdot \mathbf{E}_{i}(\mathbf{r}) d V
$$

should be a maximum. Using the expressions for $E_{p}(r)$ and $\mathbf{E}_{i}(\mathbf{r})$ as given by, ${ }^{1}$ this occurs when

$$
\bar{k}_{s} \pm \bar{k}_{p} \pm k_{i}=0 \text {. }
$$

This is the phase matching condition which will be discussed in detail in Section $\mathrm{X}$. When this condition is fulfilled the threshold condition can be approximated by

$$
E_{0}^{2} \geq \frac{64 T \epsilon}{\gamma^{2} L_{s} k_{s} L_{i} k_{i}} F
$$

where $F \sim 8 V_{l} / V_{s}$ and where we assume that the volume common to the three modes is that of the acoustic column.

\section{Frequency Conversion by Coherent \\ Scattering of Light from a Sound Wave}

Another class of interactions which can be handled by the present formalism is that of frequency conversion [10]-[12]. A phonon of frequency $\omega_{s}$ "combines" with a photon at $\omega_{p}$ to produce a new photon at the sum frequency

$$
\omega_{i}=\omega_{p}+\omega_{s} .
$$

We assume again, that the intense pump field at $\omega_{p}$ loses so little energy in the process of this interaction that its amplitude remains constant. We go back, as in Section VI, to (38), but this time keep only the terms with the time factor $\exp \left[i\left(\omega_{\nu}+\omega_{s}\right) t\right]=\exp i\left(\omega_{i} t\right)$. The result is

$$
\begin{aligned}
& \frac{d a_{i}^{+}}{d t}=i \omega_{i} a_{i}^{+}+i \eta^{*} e^{i \omega_{p} t} a_{s}^{+} \\
& \frac{d a_{s}^{+}}{d t}=i \omega_{s} a_{s}^{+}+i \eta e^{i \omega_{p} t} a_{i}^{+} \\
& \omega_{i}=\omega_{p}+\omega_{s} .
\end{aligned}
$$

Assuming a real $\eta$, which is the case when momentum is conserved, we can solve for $a_{i}^{+}$and $a_{s}^{+}$and, after taking the expectation values of the operators $n_{i}(t)=\left\langle a_{i}^{+}(t) a_{i}(t)\right\rangle$ and $n_{s}(t)=\left\langle a_{s}^{+}(t) a_{s}(t)\right\rangle$, obtain

$$
\begin{aligned}
& n_{i}(t)=n_{i 0} \cos ^{2} \eta t+n_{s 0} \sin ^{2} \eta t \\
& n_{s}(t)=n_{s 0} \cos ^{2} \eta t+n_{i 0} \sin ^{2} \eta t .
\end{aligned}
$$

If only one input is present at $t=0$, the quanta will "oscillate" back and forth between the two modes with a period of $\pi(2 \eta)^{-1}$. The total number of quanta is $n_{i}(t)+$ $n_{s}(t)=n_{i 0}+n_{s 0}$ and is a constant. There is no average power drain on the pump source. By turning off the pump after a time $\pi(2 \eta)^{-1}$ we can convert an initial acoustic input of $n_{s 0}$ phonons to an optical output of equal number of photons. This entails an energy gain of $\omega_{i} / \omega_{s}$ but, unlike the case of parametric oscillation and amplification described in Section VII, no increase in the number of quanta. This basic difference between the case of parametric down-conversion in which amplification is possible and parametric frequency-conversion, where it is not, is well known. It has been treated by a number of authors describing different physical cases [1], [13], [14]. Physically the reason for this difference is that in the case of frequency conversion the basic scattering process involves a combina- 
tion of a boson at $\omega_{p}$ with one at $\omega_{s}$ or $\omega_{i}$ to produce a boson at $\omega_{i}=\omega_{p}+\omega_{\varepsilon}$ or $\omega_{s}=\omega_{i}-\omega_{p}$. Once the supply of $\omega_{s}$ or $\omega_{i}$ bosons is depleted the production of $\omega_{i}$ or $\omega_{s}$ bosons must halt and the reverse process starts. In the down-conversion process we "split" a pump boson into an idler $\omega_{i}$ and a signal boson at $\omega_{s}$. As long as we have an inexhaustible supply of pump energy, which is the case in our model, this transfer of energy can take place, hence the continuous amplification.

Unlike the case of parametric amplification, there is no difference in this case between the quantum-mechanical solution for the expectation values $\left\langle a^{+}(t) a(t)\right\rangle$ and the classical solution for the mode energies. This is just a manifestation of the fact that parametric frequency conversion cannot be initiated by zero field vibrations.

\section{Phase Matching}

The threshold condition for the buildup of acoustic and electromagnetic oscillations as given by (56) contains the square of the "coherence" integral

$$
I=\int_{V} e^{i \mathrm{k}_{s} \cdot \mathrm{r}} \mathbf{E}_{p}(\mathrm{r}) \cdot \mathbf{E}_{i}(\mathbf{r}) d V
$$

For low threshold operation this integral must be a miximum. If we take $\mathbf{E}_{p}(\mathbf{r})$ and $\mathbf{E}_{i}(\mathbf{r})$ as standing waves along the directions $\mathbf{k}_{i}$ and $\mathbf{k}_{p}$, respectively, i.e.,

$$
\begin{aligned}
& \mathrm{E}_{p}(r)=\left(\frac{2}{V}\right)^{1 / 2} \sin \left(\mathbf{k}_{p} \cdot \mathbf{r}\right) \\
& \mathrm{E}_{i}(r)=\left(\frac{2}{V}\right)^{1 / 2} \sin \left(\mathbf{k}_{i} \cdot \mathbf{r}\right)
\end{aligned}
$$

the coherence integral is seen to contain the factor

$$
\int e^{i \mathrm{~K}_{s} \cdot r}\left(e^{i \mathrm{~K}_{p} \cdot \mathbf{r}}-e^{-i \mathbf{K}_{p} \cdot \mathbf{r}}\right)\left(e^{i \mathbf{k}_{i} \cdot \mathbf{r}}-e^{-i \mathrm{~K}_{i} \cdot \mathbf{r}}\right) d V
$$

This factor is of the order of magnitude of $1 / k$, which for optical frequencies is $\sim 10^{-5}$, unless $\mathbf{k}_{p}=\mathbf{k}_{i}+\mathbf{k}_{8}$, for which case the integral is equal to $V$, where $V$ is the volume of the common interaction region. The phase matching condition

$$
\mathbf{k}_{p}=\mathbf{k}_{i}+\mathbf{k}_{s}
$$

that must be satisfied, for low threshold, can be viewed as a statement of conservation of momentum. The onedimensional analogue of this condition was first formulated by Tien [13], extensions to three dimensions were considered by Armstrong et al. (14), and Kroll [15]. If the interaction region is limited to a distance $L$, then (63) can be violated by an amount $\left|\Delta\left(\mathbf{k}_{p}-\mathbf{k}_{i}-\mathbf{k}_{s}\right)\right| \leq 1 / L$ without an appreciable increase in threshold.

The vector relation (63) is shown in Fig. 1(a). Since the velocity of sound is, typically, a factor $\sim 10^{5}$ smaller than that of light, it follows from (63), that the maximum sound frequency that can be generated, i.e., the maximum

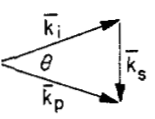

(a)

$$
\bar{k}_{p}=\bar{k}_{i}+\bar{k}_{s}
$$

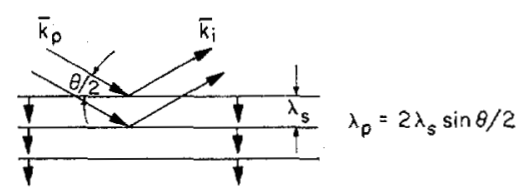

(b)
Fig. 1. (a) The phase matching vector diagram for the case of parametric amplification (and oscillation). (b) A Bragg-type diagram equivalent to (a). The sound wave is "receding" from the incident pump wave.

frequency shift, is $\sim 10^{-5} f_{p}$. We can consequently assume, to the same high degree of accuracy, that $k_{v}=k_{i}$ and obtain

$$
k_{s}=2 k_{p} \sin \frac{\theta}{2}
$$

or

$$
\omega_{p}-\omega_{i}=\omega_{\diamond}=2 \omega_{p}\left(\frac{v_{0} n}{c}\right) \sin \frac{\theta}{2}
$$

where $v_{0}=\omega_{s} / k_{s}$ is the velocity of sound in the medium, $c$ is the velocity of light in vacuum, $n$ is the refractive index, and $\theta$ is the angle between the direction of the incident pump wave and the direction of scattered idler wave.

Equation (64) was first given by Brillouin [17]. It has been used recently in the stimulated Brillouin scattering experiment of Chiao et al. [2], to determine the velocity of sound at $G c / s$ frequencies.

The stimulated sound wave travels in a direction making an angle $\frac{1}{2}(\pi-\theta)$ with the pump wave direction of propagation. The vector relationship (63) is used to draw up Fig. 1(b) in which the sound wave front is shown. The frequency shift (64) is consistent with the Doppler shift experienced by a light wave incident at the Bragg angle $\theta / 2$ on a sound wave receding with a velocity $v_{0}$ [10], [11]. The difference between the stimulated scattering [2] and earlier experiments [10], [11] is that in the former case the sound energy is not fed in from an external source but is generated by the light wave itself. The statement about the Bragg condition becomes clear if we write the first of $(64), k_{s}=2 k_{p}$ sin $\theta / 2$, as $\lambda_{p}=2 \lambda_{s} \sin \theta / 2$. The angle between the incident or scattered wave normal and the sound wave front is $\theta / 2$. The distance between equivalent scattering planes is the sound wavelength $\lambda_{\text {. }}$. The stimulated scattering that occurs once threshold is exceeded takes place in all directions. We can limit it to one direction only by lowering the losses corresponding to the wave propagating in that direction. This can be done by forming an optical resonator with an axis along $\theta$. We can, in principle, lower the threshold even further by resonating the acoustic wave in the complimentary direction $\frac{1}{2}(\pi-\theta)$. In cases where the acoustic losses are so high that the mean phonon lifetime is shorter than 


$$
\bar{k}_{i}=\bar{k}_{p}+\bar{k}_{s}
$$

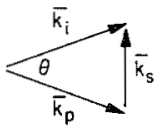

(a)

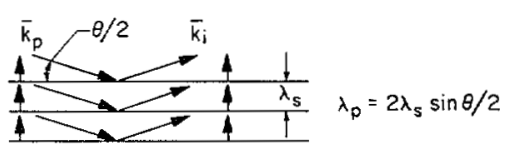

(b)
Fig. 2. (a) The phase matching vector diagram for the case of frequency conversion. (b) A Bragg-type diagram equivalent to (a). The sound wave is approaching toward the incident pump wave.

$L / v_{0}$, "resonating" the acoustic wave will not lower the threshold. A change of $\theta$ can thus afford a means of tuning the frequency shift of the scattered wave and the concomitant sound frequency. Similar considerations, for the case of light waves, have been discussed by Kroll [15].

In the frequency conversion experiment the phase matching condition takes the form

$$
\mathbf{k}_{i}=\mathbf{k}_{p}+\mathbf{k}_{\mathrm{s}}
$$

which is just a statement of conservation of momentum between the input pump photon and phonon and the output idler photon.

The phase matching vector diagram for the frequency conversion of light by sound is shown in Fig. 2(a). It is identical to the case of amplification, Fig. 1(a), except for a reversal in the direction of the sound wave. The angular relationship between the incident EM wave at $\omega_{p}$, the scattered EM wave at $\omega_{i}$, and the sound wave at $\omega_{s}$, plus the energy conservation condition $\omega_{i}=\omega_{p}+\omega_{s}$, are all consistent with Doppler shifting of the frequency of a light wave incident on an approaching sound wave at the first-order Bragg angle. Figure 2(b) is the Bragg diagram for this case.

\section{Traveling Waves Interaction}

Up to this point the treatment was devoted to interaction between resonator-type modes and was in the time domain. Another class of problems is that of the traveling wave interaction in which the distance variable, say $z$ takes the place of $t$.

We can transform from the temporal domain to the spatial domain by replacing $\omega$ by $k$ and $t$ by $z$. The equations of motion for the parametric amplification case can be written directly from (40) and (41) as

$$
\begin{aligned}
& \frac{d a_{i}^{+}}{d z}=i k_{i} a_{i}^{+}-i \eta e^{i k_{p z}} a_{s} \\
& \frac{d a_{s}}{d z}=-i k_{s} a_{s}+i \eta^{*} e^{-i k_{p 2}} a_{i}^{+}
\end{aligned}
$$

where

$$
\eta \sim\left(\frac{\gamma}{64 \epsilon T}\right)^{1 / 2}\left(k_{i} k_{s}\right)^{1 / 2} E_{\text {pump }}
$$

The quantity $a_{i}^{+} a_{i}$ is now proportional to $P_{i} / \omega_{i}$, i.e., classically, to the boson flux so that the Manley-Rowe relations are again satisfied. The solutions of (66) can be taken directly from (42) through (47).

In a similar way we can write the equations for frequency conversion by transforming (59). The result is

$$
\begin{aligned}
& \frac{d a_{i}^{+}}{d z}=i k_{i} a_{i}^{+}+i \eta{ }^{\prime \prime} e^{i k_{p z} z} a_{s}^{+} \\
& \frac{d a_{s}^{+}}{d z}=i k_{s} a_{s}^{+}+i \eta e^{i k_{p} z} a_{i}^{+} .
\end{aligned}
$$

The solutions are obtained directly from Section IX.

\section{Summary}

The problem of interaction of light and hypersound has been considered. The interaction is provided by a perturbation Hamiltonian which describes the change in electrostatic energy due to the presence of acoustic waves. Specific solutions were obtained for the cases of two EM and one sound modes. Losses were included phenomenologically in order to obtain the threshold condition for stimulated Brillouin scattering. The relation between the quantum-mechanical and classical solutions is examined and except for presence of zero-field terms in the case of parametric amplification they lead to the same results.

\section{References}

[1] Louisell, W. H., A. Yariv, and A. E. Siegman, Quantum fluctuations and noise in parametric processes, I, Phys. Rev., vol 124, Dec 15, 1961, pp 1646-1654.

[2] Chiao, R. Y., C. H. Townes, and B. P. Stoicheff, Stimulated Brillouin scattering and coherent generation of intense hypersonic waves, Phys. Rev. Letters, vol 12, May 25, 1964, pp 592595. [3] Garmire, E., and C. H. Townes, Stimulated Brillouin scattering
in liquids, Appl. Phys. Letters, vol 5, Aug 15, 1964, pp 84-86.

[4] Louisell, W. H., Coupled Modes and Parametric Electronics. New York: Wiley, 1960.

[5] Slater, J. C., Microwave Electronics. Princeton, N. J.: Van Nostrand, $1960, \mathrm{p} 57$.

[6] Kittel, C., Quantum Theory of Solids. New York, London: Wiley, 1963.

[7] Gerber, R., and C. H. Townes, Quantum Electronics-A Symposium, C. H. Townes, Ed. New York: Columbia University Press, $1960, \mathrm{p} 247$.

[9] Kingston, R. H., Parametric amplification and oseillation at optical frequencies, Proc. IRE (Correspondence), vol 50, Apr $1962, \mathrm{p} 472$.

[10] Siegman, A. E., C. F. Quate, J. Bjorkholm, and G. Francois, Frequency translation of an He-Ne laser's output frequency by acoustic output coupling inside the resonant cavity, $A p p l$. Phys. Letters, vol 5, Jul 1, 1964, pp 1-2.

[11] Cummins, H. Z., and N. Knable, Single sideband modulation of coherent light by Bragg reflection from acoustical waves, Proc. IEE (Correspondence), vol 51, Sep 1963, p 1246.

[12] Quate, C. F., H. J. Shaw, C. D. W. Wilkinson, and D. K. Winslow, $J$. Appl. Phys., to be published.

[13] Korpel, A., R. Adler, and B. Alpiner, Stimulated Brillouin seattering in liquids, Appl. Phys. Letters, vol 5, Aug 15, 1964, pp 86-88.

[14] Tien, P. K., Parametric amplification and frequency mixing in propagating circuits, $J$. Appl. Phys., vol 29, Sep 1958, pp $1347-1357$.

[15] Armstrong, J. A., N. Bloembergen, J. Ducuing, and P. S. Pershan, Interactions between light waves in a nonlinear dielectric, Phys. Rev., vol 127, Aug 15, 1962, pp 1918-1939.

[16] Kroll, N. M., Parametric amplification in spatially extended media and application to the design of tuneable oscillators at optical frequencies, Phys. Rev., vol 127, Aug 15, 1962, pp $1207-1211$.

[17] Brillouin, L., Diffusion de la lumiere et des rayon $x$ par un corps transparent homogene, Annales de Physique (Paris), vol 17,1922 , pp 88-122. 